\title{
Psychological services for women giving birth in a large Irish tertiary referral maternity unit
}

\author{
Aileen McAuliffe, ${ }^{1}$ Noirin E. Russell, ${ }^{1}$ Joanne Fenton ${ }^{1}$
}

The Psychiatrist (2011), 35, 92-94, doi: 10.1192/pb.bp.109.029165

${ }^{1}$ Coombe Women and Infants University Hospital, Dublin, Ireland Correspondence to Aileen McAuliffe (ailinmcauliffe@gmail.com)

First received 16 Dec 2009, final revision 10 Jun 2010, accepted 22 Jul 2010

\begin{abstract}
Aims and method To investigate whether women were being asked about their current and previous mental health, and where risk factors for perinatal mental health problems were identified, to determine whether appropriate referral occurred. Individual case-note review was performed on women where present or past mental health problems were indicated.

Results In two-thirds of women where a history of mental health problems was indicated at the booking clinic this was not addressed in their obstetric case notes. Of the women who reported being depressed, nearly a third had no reference to this in their obstetric case notes and only 40\% were referred to the Mental Health Liaison Clinic (MHLC). Two-thirds of the women who indicated a history of postnatal depression had no further comments documented in their obstetric case notes. One case of bipolar affective disorder and one case of puerperal psychosis were identified on booking questionnaires and neither had any comments documented in the obstetric case notes. In total $16 \%$ of women who reported a history of mental health problems were referred to the MHLC.
\end{abstract}

Clinical implications More robust procedures for the identification and management of perinatal mental health risk need to be implemented.

Declaration of interest None.
Perinatal mental health is a major public health concern, with $10 \%$ of postpartum mothers developing a depressive illness in the first year following delivery. ${ }^{1}$ Four per 1000 will be admitted to a psychiatric unit, 2 per 1000 with puerperal psychosis. ${ }^{2}$ Postnatal depression is the most common peripartum mental health problem, with an incidence of $13-20 \% .^{3}$ When untreated, it can result in partner depression, higher divorce rates, higher rates of behavioural problems in children and lower emotional and cognitive developmental scores in children. ${ }^{4}$

The Confidential Enquiries into Maternal Deaths (CEMD) highlight the high human cost of perinatal mental illness, with suicide the leading cause of maternal death in the 2000-2002 report. ${ }^{5}$ The last CEMD (2003$2005)^{6}$ showed a decline in numbers of deaths by suicide, possibly owing to the implementation of improvements suggested in the 2000-2002 report, but psychiatric illness is still responsible for 0.85 deaths per 100000 pregnancies, equivalent to the number of deaths owing to pre-eclampsia and eclampsia. Psychiatric disease is responsible for $6 \%$ of maternal mortality in the $\mathrm{UK}^{6}$ and it has been a consistent finding over the past decade that the risk of recurrence of psychiatric disorder is poorly identified and managed. Sixty per cent of women who took their own life within the first year postpartum had a history of serious mental illness but only $40 \%$ of these were identified antenatally and only $20 \%$ had a detailed management plan in place.

In Ireland there are three dedicated mental health liaison clinics (all in Dublin), where perinatal mental healthcare is provided on a sessional basis by consultant psychiatrists with a special interest in perinatal mental health. In the remainder of the country, care is provided by general adult psychiatrists on an on-call basis. One of the main differences in the provision of antenatal care in Ireland when compared with the UK is that traditionally the majority of Irish women are seen regularly by an obstetrician during their pregnancy. However, in recent years there has been an increase in midwifery-led antenatal care and shared care between the primary care physician and the midwife.

The aim of this study was to determine the level of psychological service provision in the Coombe Women and Infants University Hospital, through review of the case notes of women who indicated in their booking questionnaire that they were experiencing, or had experienced in the past, mental health problems. Where mental health problems were identified by the midwife, we aimed to assess whether the obstetrician examined the patient's mental state, whether their mental health history was documented in the medical notes and if so, whether they were referred to 
the hospital social worker, to the mental health liaison clinic or to their sector mental health team.

\section{Method}

The study took place in the Coombe Women and Infants University Hospital, which has 8000-9000 deliveries per year. Women are assessed initially at the midwife booking clinic, where they complete a booking questionnaire which includes questions on obstetric and surgical history, medical and psychiatric history, as well as drug and social history. All women are thereafter assessed by an obstetrician at the obstetrician first visit clinic. There were 8088 deliveries in our hospital in 2006, with 7937 booking questionnaires completed. A sample of women who both booked and delivered in 2006 was chosen $(n=4188)$. Individual casenote review was performed on $27.4 \%$ of women (1147/4188) where past or present mental health problems were indicated. Patients with a standalone diagnosis of eating disorder, counselling or panic $(n=648)$ were excluded as the aim of the investigation was to focus on severe and enduring mental illness. These patients were included if they had a comorbid illness. Of the 499 women with a significant mental health history, $95 \%$ of the charts were available for review.

\section{Results}

When the case notes were reviewed, $12 \%(n=499 / 4188)$ of women had a significant mental health history. Figure 1 details the information obtained at this initial midwifery booking visit.

Figure 2 summarises the documentation of mental health issues in the obstetrician's notes during the pregnancy. In $66 \%$ of women with a history of mental health problems $(n=328)$ there was no documented comment in the obstetric notes. There were 69 referrals to the mental health liaison clinic during the period under study. Of the 47 women who reported feeling depressed at the time of filling out the booking questionnaire, $26 \%$ had no comment made by the obstetrician in their case notes

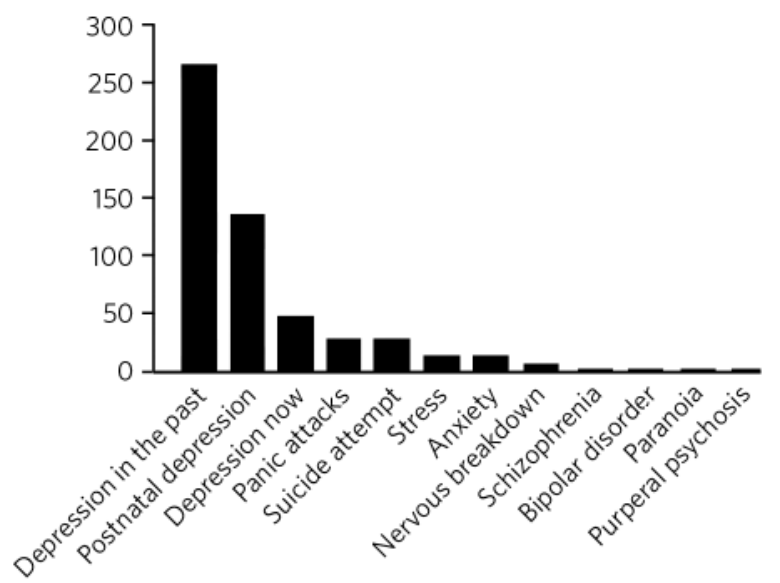

Fig 1 Mental health information on booking questionnaire $(n=499)$. and only $40 \%$ were referred to the mental health liaison clinic. Of the 135 women who reported a history of postnatal depression, in $63 \%$ of cases this was not addressed by the obstetrician and only $16 \%$ were referred to the mental health liaison clinic. One patient reported a history of bipolar affective disorder, one a history of puerperal psychosis and one a history of schizophrenia. There was no comment documented in the obstetric case notes of those patients, with no referral to psychiatric services or no evidence of a detailed multidisciplinary management plan.

\section{Discussion}

Forty-seven women reported being depressed when completing the booking questionnaire. Out of a total of 4188 women, this is a low rate (1\%). In the 2001 European Outcome of Depression International Network (ODIN) study, Ayuso-Mateos et $a l^{7}$ reported the prevalence of female depressive episodes in urban Ireland at $20 \%$ (95\% CI 5.3-53.9).

The rate of bipolar affective disorder and schizophrenia in our study was 1 reported patient out of 4188. These low rates could possibly be explained by a number of reasons women being reluctant to report illness themselves because of stigma, the fear of Social Services becoming involved or inflexible phraseology in the booking questionnaire, which may make it difficult for the woman to report her diagnosis. It is possible that lack of insight into their illness may also account for the low rates of reported mental illness in our study. It is also possible that the retrospective design of the study may lead to falsely low rates, whereas a prospective, specifically designed questionnaire may provide a more accurate picture. We agree with the view that the blanket term 'postnatal depression' is unhelpful and may do a disservice to women experiencing severe postnatal episodes of illness. ${ }^{8}$

\section{Strengths and limitations}

Ninety-five per cent of the charts were available for review. The hospital's electronic database was used to identify cases; however, we acknowledge that data entry into the

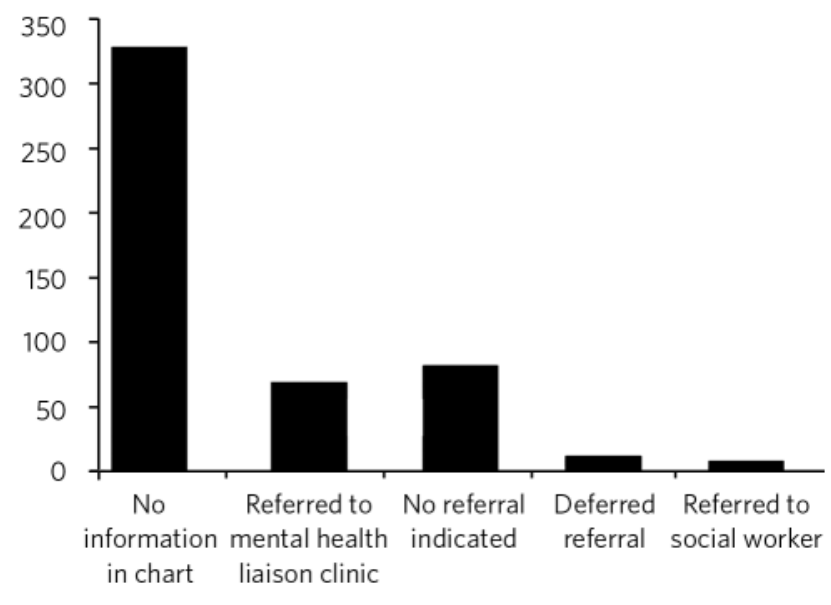

Fig 2 Documentation of psychiatric history in obstetric medical notes. 
database may have been subject to entry error. This investigation used only the written case notes as evidence that the obstetrician addressed the mental health history. In some cases there may have been conversations in relation to the mental health history which were not documented. Likewise, there may have been verbal referrals to the social worker or sector mental health teams that were not documented. These findings are based on an Irish setting, therefore it is difficult to estimate how generalisable they are to other settings.

\section{Further actions}

The results of this study were presented at the grand rounds in the Coombe Women and Infants University Hospital, and the response from the consultants and midwives was very positive. We have been invited to take part in the midwife educational training programme and have recommended that the non-consultant hospital doctor induction programme should incorporate a module on perinatal mental health. This should include practical interview skills so as to make it easier for obstetric staff to enquire about mental health concerns. Also recommended is the appointment of a dedicated mental health liaison nurse on site to provide immediate advice and support. We are happy to report that a dedicated mental health liaison nurse commenced practice in June 2010. As a result of the findings from our study, a mental health liaison clinic referral form was designed to make it easier for hospital staff to refer patients to our service. Future initiatives include a poster campaign in the hospital to increase awareness of psychological problems and reduce stigma and the compilation of an information booklet on perinatal mental health which will be given to all patients at their booking clinic visit.

\section{Acknowledgements}

We thank the Master of the Coombe Women and Infants University Hospital, the Medical Ethics Committee and the IT and Medical Records Department.

\section{About the authors}

Aileen McAuliffe, Senior Registrar in Psychiatry, Noirin E. Russell, Specialty Registrar in Obstetrics and Gynaecology, and Joanne Fenton, Consultant Psychiatrist, Coombe Women and Infants University Hospital, Dublin, Ireland.

\section{References}

1 Cox JL, Holden JM, Sagovsky R. Detection of postnatal depression. Development of the 10-item Edinburgh Postnatal Depression Scale. $\mathrm{Br}$ Psychiatry 1987; 150: 782-6.

2 Kendell RE, Chalmers JC, Platz C. Epidemiology of puerperal psychoses. Br J Psychiatry 1987; 150: 662-73.

3 Cox JL, Murray D, Chapman G. A controlled study of the onset, duration and prevalence of postnatal depression. Br J Psychiatry 1993; 163: 27-31.

4 Lyons-Ruth K, Zoll D, Connell D, Grunebaum HU. The depressed mother and her one-year-old infant: environment, interaction, attachment and infant development. In Maternal Depression and Infant Disturbance: New Directions for Child Development (eds EZ Tronick, T Field): 34. JosseyBass, 1986.

5 Lewis G, Confidential Enquiry into Maternal and Child Health. Why Mothers Die (2000-2002): The Sixth Report of the Confidential Enquiries into Maternal Deaths in the United Kingdom. RCOG Press, 2004.

6 Confidential Enquiry into Maternal and Child Health. Saving Mothers Lives: The Seventh Report of the Confidential Enquiries into Maternal Deaths in the United Kingdom. CEMACH, 2007.

7 Ayuso-Mateos JL, Vázquez-Barquero JL, Dowrick C, Lethinen V, Dalgard OS, Casey $P$, et al. Depressive disorders in Europe: prevalence figures from the ODIN study. Br J Psychiatry 2001; 179: 308-16.

8 Oates M. Postnatal affective disorders. Part 1: An introduction. Obstetrician Gynaecologist 2008; 10: 145-50. 